\title{
Nodular Sclerosis Classic Hodgkin Lymphoma by Ann Arbor Stage
}

National Cancer Institute

\section{Source}

National Cancer Institute. Nodular Sclerosis Classic Hodgkin Lymphoma by Ann Arbor

Stage. NCl Thesaurus. Code C141223.

An anatomic stage for nodular sclerosis classic Hodgkin lymphoma based on the Ann Arbor classification criteria. 